\title{
Deleterious MSH2 Gene Mutation
}

National Cancer Institute

\section{Source}

National Cancer Institute. Deleterious MSH2 Gene Mutation. NCI Thesaurus. Code C131777.

A change in the nucleotide sequence of the MSH2 gene that is associated with increased risk of disease. 